\title{
Les cartes postales dans les stations de sports d'hiver haut de gamme : du produit touristique à l'enjeu de communication
}

Piquerey Lise

\section{(2) OpenEdition} Journals

Édition électronique

URL : http://journals.openedition.org/tourisme/1113

DOI : 10.4000/tourisme.1113

ISSN : 2492-7503

Éditeur

Éditions touristiques européennes

Référence électronique

Piquerey Lise, « Les cartes postales dans les stations de sports d'hiver haut de gamme: du produit touristique à l'enjeu de communication », Mondes du Tourisme [En ligne], Hors-série | 2016, mis en ligne le 01 septembre 2016, consulté le 01 mai 2019. URL : http://journals.openedition.org/tourisme/1113 ; DOI : 10.4000/tourisme.1113

Ce document a été généré automatiquement le 1 mai 2019.

\section{c) $(1) \ominus$}

Mondes du tourisme est mis à disposition selon les termes de la licence Creative Commons Attribution - Pas d'Utilisation Commerciale - Pas de Modification 4.0 International. 


\title{
Les cartes postales dans les stations de sports d'hiver haut de gamme: du produit touristique à l'enjeu de communication
}

\author{
Piquerey Lise
}

1 Les cartes postales, objets emblématiques associés aux périodes de vacances, sont des marqueurs spatiaux des lieux touristiques, dans le sens où elles offrent une représentation socialement définie de ces derniers, ou plutôt d'une portion choisie de ces derniers. Le rôle de cet article n'est pas de dresser un historique de la création puis de l'expansion des cartes postales, mais plutôt de questionner cet outil de diffusion de l'imaginaire touristique. Les cartes postales peuvent-elles être perçues comme des vecteurs promotionnels d'une destination touristique? Les ouvrages parus sur les stratégies marketing dans le champ du tourisme placent en premier lieu la communication touristique comme étant le procédé ultime pour mettre en avant «l'image » des lieux touristiques. Les outils proposés, bien que très variés, n'invoquent pas la carte postale. "L'image » se définit comme «la somme agrégée des croyances, idées, impressions et attentes qu'un touriste a à propos d'une destination touristique " (Crompton, cité dans Frochot et Legohérel, 1997, p. 277). L'image, élément hautement subjectif, est donc une construction certes propre à chacun, mais issue de constructions sociales, régie par et pour des individus ayant des caractéristiques socio-économiques identiques, ou du moins similaires. Les cartes postales sont conditionnées par un procédé de fabrication centré sur l'individu. Ce dernier sera le référentiel direct en tant que client lors de la prise de vue et de la constitution de la carte postale qui doit convenir à ses goûts, afin qu'il ne soit plus placé comme réceptacle de la construction, mais bien comme actant, en achetant la carte postale. Il sera ensuite un vecteur de l'imaginaire touristique lorsqu'il choisira de l'envoyer à un destinataire, référentiel indirect du choix de la carte postale lors de l'achat. Dans cette logique, la carte postale instaure son rôle de marqueur social. L'idée de marqueur, en tant qu'objet spécifique appliqué à un objectif précis, 
permet de leur procurer une tridimensionnalité : à la fois spatiale, sociale et temporelle. En effet l'image, abstraite ou non d'un espace, prise à un temps donné, se veut être représentative de la période dans laquelle s'inscrit la communication de la destination touristique. Le caractère spatial de la carte postale est intrinsèquement présent dans sa construction sociale et temporelle, puisqu'il permet de distinguer la destination de l'ensemble du champ touristique à travers ses caractéristiques de sites. Ces dernières sont mises en scène par les prises de vue. Le paysage évolutif, bien que perçu à travers l'œil du photographe, se fige pour se transformer en carte postale.

2 L'image de la carte postale, au même titre que les images présentes dans les brochures ou dans d'autres outils de communication touristique, est une carte de visite pour la destination, dans le sens où elle doit en donner le meilleur aperçu possible (Piquerey, 2012). L'image, définie comme la représentation d'un objet, doit donc «singulariser la notoriété aux yeux du public» (Le Petit Robert, 2011, p.1276) et tendre à promouvoir l'image de marque de cette destination. Si cela se construit à travers le jeu du texte et de l'image dans les brochures touristiques, les cartes postales, à "vue simple », "vue multiple ", avec ou sans texte, sont aussi des vecteurs de l'image grâce à leur objectif de diffusion des acteurs du lieu. L'image présente sur les cartes postales peut donc être apparentée à un des outils permettant au lieu de construire son identité par rapport et en référence à l'ailleurs et à autrui (Castra, cité dans Paugam, 2010, p. 72), compris ici comme l'ensemble des autres destinations touristiques. Cette identité spatiale est double : à la fois identité de lieu, ses traits physiques ou idéologiques lui procurant la capacité d'être distingué par les individus (Moles, cité dans Bailly, Ferras et Pumain, 1992, p. 191) et à la fois identité spatiale, dans la relation autoconstructive qu'entretient un individu avec ce lieu, potentiellement influencé par l'ensemble « des discours, des représentations et des pratiques normatives du bon usage de son espace par un groupe donné » (Jacques Lévy, dans Lévy et Lussault, 2003, p. 480).

L'image, en tant que construit social normé, ne peut-elle pas s'avérer davantage image d'Épinal ou de marque de la destination qu'image réelle ? Comme le souligne Jean-Baptiste Litot, « de façon comparable à une marque ou un produit, le territoire doit se distinguer pour exister à partir d'attributs et de valeurs qu'il souhaite transmettre, il faut se créer une image facilement identifiable et reconnaissable » (2010, p. 64). À la manière d'un logo qui permet d'identifier un élément par un symbole, la carte postale ne permet-elle pas d'identifier des territoires avec des éléments récurrents, qui se placeraient comme des emblèmes territoriaux? L'emblème territorial, «fraction d'un espace, en général un lieu ou/et un monument, qui, par métonymie, représente et même signifie cet espace et les valeurs qui lui sont attribuées » (Ruby, cité dans Lévy et Lussault, 2003, p. 305), n'est-il pas un outil de création puis d'affirmation d'une idéologie spatiale? C. Ruby utilise la définition de Gilbert (1986) pour mettre en avant le rôle fédérateur des idéologies au sein d'un groupe: «système d'idées et de jugements, organisé et autonome qui sert à décrire, expliquer, interpréter ou justifier la situation d'un groupe ou d'une collectivité dans l'espace » (cité dans Lévy et Lussault, 2003, p. 482). Les idéologies sont donc des systèmes de pensées intentionnels, construits pour s'appliquer à un espace et à destination d'un groupe d'individu. En reprenant cette hypothèse pour les cartes postales, ne seraientelles pas des constructions socio-culturelles, mettant en avant des emblèmes territoriaux dans l'optique de participer à la création de l'imaginaire touristique propre aux stations de sports d'hiver, à destination des touristes? 
Dès lors, la définition de l'idéologie spatiale entre en résonance avec la notion d'identité sociale. Bernard Debarbieux définit l'identité sociale comme étant le "produit de l'identification d'un groupe par des individus qui lui sont extérieurs [...] sur la base d'une série d'identifiants dont certains sont culturels (langue, pratique religieuse, coutumes, etc.) et qui permet de situer le groupe dans une représentation d'ensemble de la société " (2008, p.93). L'imaginaire socio-spatial de la montagne pourrait donc être une construction exogène aux espaces montagnards, propre à des individus extérieurs les identifiant comme relevant de «la montagne». L'appropriation de ces représentations par les populations résidentes a permis d'élire ces espaces au rang d'emblèmes territoriaux, vecteurs d'une idéologie socio-spatiale qui les unit et les identifie par rapport au reste de la société. Ainsi, l'identité sociale se retranscrit à partir d'éléments spatiaux au sein des stations de sports d'hiver. Ces éléments, choisis par des acteurs touristiques, présents ou non, développent ce que nous pouvons appeler une représentation de la montagnité, définie comme l'ensemble des éléments mis en scène dans les communications et les représentations produites par les acteurs du système touristique de montagne, et qui peuvent être érigés, ou non, au rang d'emblèmes territoriaux. Ce terme est construit en référence à la notion d'italianité développée par Roland Barthes (1964). Dans le cadre d'une analyse des éléments qui composent une publicité Panzani, Roland Barthes développe l'idée que ces éléments ne représentent pas l'Italie, mais l'italianité dans le sens où ils recréent un imaginaire territorial en mettant en avant les caractéristiques italiennes du produit proposé. Ces caractéristiques évoquent l'Italie aux individus, grâce à leurs bagages socio-culturels. Ainsi les outils de communication des stations de sports d'hiver appréhenderaient la mise en image de leur territoire à travers le filtre de la montagnité, ce qui leur permettrait de se rendre accessibles au plus grand nombre. Les cartes postales, et leur utilisation d'une certaine représentation de la montagnité, sont donc potentiellement des vecteurs de l'imaginaire touristique et de l'identité sociale des stations de sports d'hiver. La construction puis la diffusion de l'imaginaire permet de donner « sens non seulement aux lieux géographiques abstraits, mais aux pratiques qui s'y développent » (Bozonnet, 1992, p. 123). Quel est le rôle des cartes postales dans la production et la diffusion de la montagnité des stations de sports d'hiver haut de gamme en Autriche, en France et en Suisse? Les cartes postales, inscrites dans les pratiques populaires et traditionnelles des périodes de vacances, sontelles, au sein d'espaces touristiques singuliers, représentatives de leur caractère haut de gamme et de la sélectivité sociale qui s'y tient?

5 Après avoir défini le caractère haut de gamme et sélectif des stations de sports d'hivers de l'échantillon, nous questionnerons la place occupée par les cartes postales dans la construction de l'identité des stations étudiées, notamment par une étude de cas basée sur un échantillonnage des cartes postales commercialisées à Megève.

\section{Être accessible et se rendre accessible : les critères de sélection des stations de sports d'hiver haut de gamme}

6 Cette étude expérimentale sur le lien entre les cartes postales et les espaces touristiques s'inscrit dans les stations haut de gamme de l'arc alpin. Le terme «haut de gamme» permet d'ores et déjà d'affilier ces territoires à ceux des élites, dans le sens où les 
infrastructures présentes seront accessibles seulement à une minorité d'individus qui a été élue. «Élue » dans le sens où, d'après le processus sélectif-électif de création des élites, les individus pouvant accéder à ces biens et ces services ont été jugés aptes par leur pairs, au vu de leurs capacités financière, sociale et culturelle.

7 La sélection des stations de sports d'hiver pouvant être identifiées comme haut de gamme se base sur deux critères principaux :

- L'orientation vers le luxe: quel est le lien entre les infrastructures haut de gamme et la clientèle? L'implantation des services marchands, qu'ils soient hôteliers ou commerciaux, répond à une étude de marché, ce qui induit l'hypothèse que si des marques de luxe et des hôtels cinq étoiles s'établissent dans les stations de sports d'hiver, c'est qu'elles accueillent une clientèle adaptée. Cette orientation s'appuie sur deux indicateurs permettant de différencier les stations de sports d'hiver de l'arc alpin. Premièrement, celui de l'hôtellerierestauration qui recense les hôtels cinq étoiles et les palaces (France) ou les hôtels cinq étoiles superior (Autriche et Suisse) ainsi que les restaurants primés au guide Michelin. Le second indicateur recense le nombre de boutiques des cinq marques de luxe jugées les plus influentes d'après le bureau d'études Interbrands (2008) : Louis Vuitton, Gucci, Chanel, Rolex et Hermès.

- L'accessibilité: ici l'accessibilité est une notion plus large que ce qu'elle revêt communément: «offre de mobilité, ensemble des possibilités effectives pour relier deux lieux par un déplacement" (Jacques Lévy, cité dans Lévy et Lussault, 2003, p. 35). L'accessibilité physique des lieux à travers le franchissement de la distance euclidienne est cependant une des modalités de cet axe de sélection, puisque le déplacement, temps mort lors de la période de vacances, ne sera pas perçu de la même manière en fonction des modes de transport. C'est également un moment du voyage durant lequel les individus sont inégaux dans leur capacité à s'affranchir de la distance plus ou moins rapidement en fonction de leur solvabilité et des infrastructures mises à disposition de la clientèle par les acteurs touristiques des stations de sports d'hiver (altiport, place d'atterrissage, gare routière, gare ferroviaire). Dans ce travail, l'accessibilité correspond également à la capacité des individus à accéder et à trouver au sein des stations de sports d'hiver des offres socio-culturelles, à travers des événements qui correspondent à leurs attentes, par exemple. Les événements proposés pendant les saisons touristiques assurent une double fonction: ils sont à destination d'un public visé qui est déjà présent ou que la station souhaite attirer, et ils permettent également de placer la station organisatrice dans une aire culturelle définie par le type d'événement qu'elle organise. Un recensement des tournois de polo, sur neige ou non, ainsi que la présence de golfs dix-huit trous, a donc été effectué pour mettre en lien les événements et les espaces élitistes. Enfin, l'accessibilité financière est un critère important pour définir ce qui permet à ces espaces touristiques de se différencier des autres. En effet, le prix d'une chambre dans un hôtel cinq étoiles ou celui des forfaits de remontées mécaniques sont des indicateurs qui permettent de juger de la perméabilité des offres de biens et de services touristiques à l'égard des catégories socio-économiques inférieures.

8 Ces deux axes principaux et les critères qui les composent permettent d'identifier un groupe de neuf stations haut de gamme au sein de l'arc alpin : Megève, Courchevel et Vald'Isère pour la France ; Gstaad, Crans-Montana, Saint-Moritz, Zermatt et Davos pour la Suisse ; Kitzbühel pour l'Autriche.

9 Ces stations haut de gamme, en accueillant des catégories socio-économiques jugées élitistes par le reste de la société, utilisent les codes et les normes de ces groupes sociaux dans leur développement et leur aménagement. Pour Nicolas Hossard (2005, p. 46) : 
L'illustration d'une carte postale se veut surtout représentative d'un lieu [...] cette représentativité est relatée par le cadre «naturel» (un paysage alentour), le cadre "culturel » (un décor urbain) comprenant également les monuments, le cadre « social » (l'aspect humain, qu'il soit oisif, visiteur ou sportif, ou autochtone, en tant que simple passant-figurant ou en tant que sujet principal de l'illustration).

De ce point de vue, comment les stations de sports d'hiver élitistes, qui puisent leur particularité dans les clientèles qu'elles accueillent, peuvent-elles produire des images et des représentations en lien avec le souci de discrétion que recherche une majeure partie de ce type de population? Plus qu'habiter ces lieux, les élites officient également dans l'aménagement de la station touristique, qu'elle soit hivernale comme Megève ou balnéaire comme Deauville, construites toutes deux par et pour la bourgeoisie et l'aristocratie. Dans les espaces qu'elles investissent, les catégories socio-économiques supérieures reproduisent à la fois des codes architecturaux propres à leur espace de résidence permanent, mais également les codes sociaux qu'elles y trouvent. À l'image de l'entre-soi résidentiel pratiqué dans leurs espaces du quotidien, les catégories socioéconomiques supérieures (re)créent un espace de l'entre-soi au sein des lieux de villégiature (Pinçon et Pinçon-Charlot, 2007, 1994). L'entre-soi se définit comme la cohésion sociale d'un groupe d'individus, produite à partir d'une homogénéité de capital à la fois économique, social et culturel. Il se matérialise à travers des marqueurs spatiaux qui délimitent les espaces privés (outils de fermeture et de sécurisation des lieux de résidences, hôteliers ou non) et, au sein des espaces publics, à travers des espaces réservés aux élites, fréquemment appelés « espaces VIP » (very important persons), qui permettent aux catégories socio-économiques supérieures de se distinguer. Des cas de privatisation d'espaces publics ont également été relatés par les acteurs rencontrés, comme par exemple des privatisations de pistes de ski pour une demi-journée ou une journée. Si les logiques du visible et du caché des catégories socio-économiques supérieures s'opèrent au sein des stations de sports d'hiver haut de gamme, quelles sont les images utilisées par les acteurs du système touristique et les éditeurs de cartes postales pour vendre un produit touristique à des fins de diffusion? Les cartes postales présentes au sein des espaces touristiques haut de gamme sont-elles vectrices des codes sociaux et culturels des élites?

\section{Les lieux de vente des cartes postales, révélateurs de la place sociale des clientèles ciblées}

$11 \mathrm{Au}$ sein des stations de sports d'hiver haut de gamme, les lieux de vente des cartes postales semblent être choisis en fonction de la visibilité des commerces. Dans l'ensemble des stations de l'échantillon, nous pouvons retrouver des espaces de vente communs (fig. 1): les rues touristiques et commerçantes principales, dans les boutiques de souvenirs, avec une plus grande fréquence de ce type de commerces dans les stations françaises et à Zermatt. Le cas de Zermatt et du Cervin semble être l'archétype de la construction d'un haut lieu à travers la mise en image d'un emblème spatial. En effet, le Cervin est un emblème à la fois pour la station de Zermatt, pour la nation helvétique et, à l'échelle internationale, en tant qu'espace privilégié pour la pratique de l'alpinisme ; son utilisation dans l'imagerie populaire et touristique se trouve donc être surexploitée (Hostettler, 2006, 1990). À proximité des rues touristiques et commerçantes, se trouvent des rues périphériques, qui articulent les flux entre la rue commerçante et les zones 
résidentielles. Dans ces zones, les cartes postales sont vendues dans les supérettes comme par exemple les magasins Coop et Migros pour la Suisse ; Sherpa, Carrefour Montagne et Petit Casino en France, ainsi que dans les agences postales. Elles se localisent à proximité des caisses et en petit nombre. Un troisième type de point de vente est présent seulement en Autriche et en Suisse: les gares ferroviaires (aucune des stations françaises de l'échantillon n'est accessible directement en train). Dans chaque gare, les traditionnels kiosques et bars sont présents, avec une prédominance de la chaîne Relay. L'ensemble de ces points de vente ont comme dénominateur commun d'être des lieux ouverts au public, à tous les types de publics. Nous pourrions donc émettre l'hypothèse que les points de vente des cartes postales recherchent avant tout une visibilité maximum. Cette dernière permet une meilleure diffusion des emblèmes touristiques et donc de la montagnité. Cependant, à la différence des boutiques de souvenirs et des supérettes, les gares sont des lieux de passage. Les individus les traversent quelles que soient leurs caractéristiques socio-économiques ou culturelles.

Fig. 1. Modèle des stratégies de localisation des lieux de vente des cartes postales dans les stations de sports d'hiver haut de gamme.

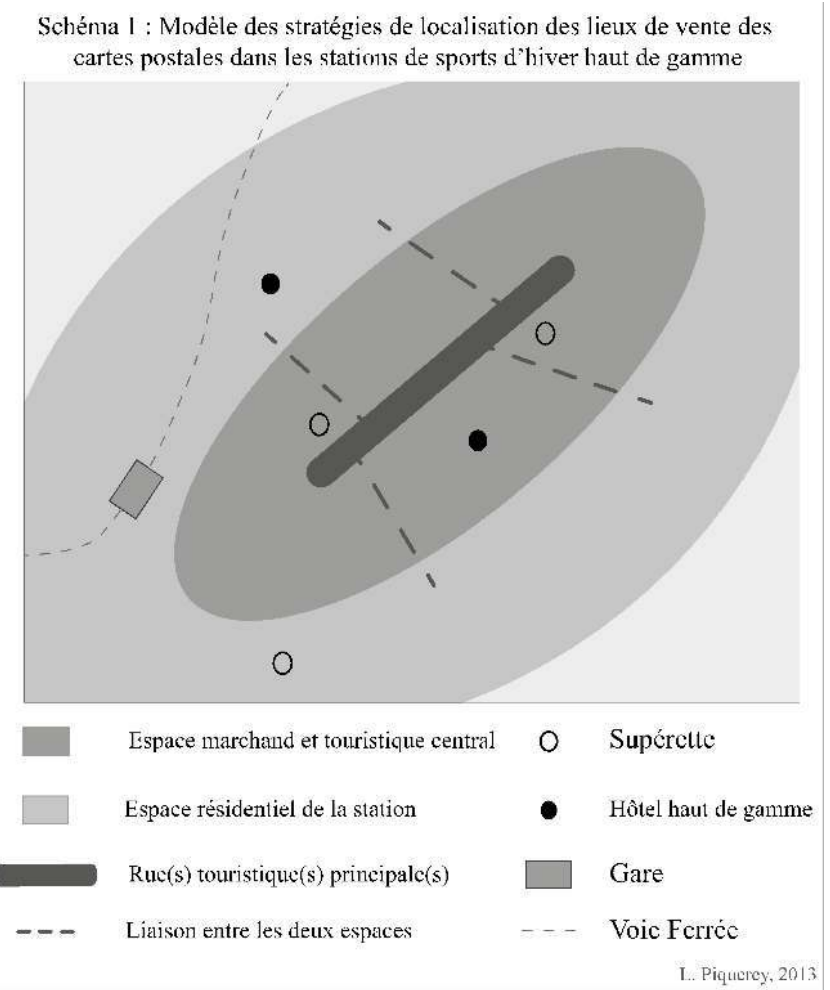

L. Piquerey, 2013.

Or, à partir d'une observation des pratiques socio-spatiales au sein des gares des stations autrichiennes et suisses lors des séjours de terrain, nous pouvons émettre l'hypothèse que la place des cartes postales dans les gares ferroviaires est corrélée aux pratiques spatiales des catégories socio-économiques. Le schéma 2 (fig. 2) permet de présenter un modèle d'organisation des gares des stations autrichiennes et suisses de l'échantillon. Les touristes qui voyagent avec des packages touristiques ou qui résident dans des hôtels haut de gamme (quatre à cinq étoiles superior) sont pris en charge dès le pied posé sur le quai. Le personnel (chauffeur ou hôtesse de tour opérateur) accueille les clients sur les 
quais ou dans le hall de gare, des voitures stationnées à proximité du parvis ou parfois directement sur ce dernier attendent les clients pour les diriger vers les lieux d'hébergement. À la fin des séjours, les clients seront pris en charge jusqu'au moment où ils monteront dans le train. Ces types de clientèles passent donc d'un mode de transport à l'autre pour ainsi optimiser les temps de déplacement grâce à une baisse de la durée des ruptures de charge. Contrairement à ces dernières, les clientèles qui résident dans d'autres types d'infrastructures (location, hôtel de une à trois étoiles) ont un temps de rupture de charge plus important, car elles ne profitent d'aucune prise en charge par un agent du système touristique, ce qui permettra d'avoir du «temps disponible » pour se déplacer dans la gare ferroviaire.

Fig. 2. Types de touristes et pratiques spatiales des gares ferroviaires des stations de sports d'hiver.

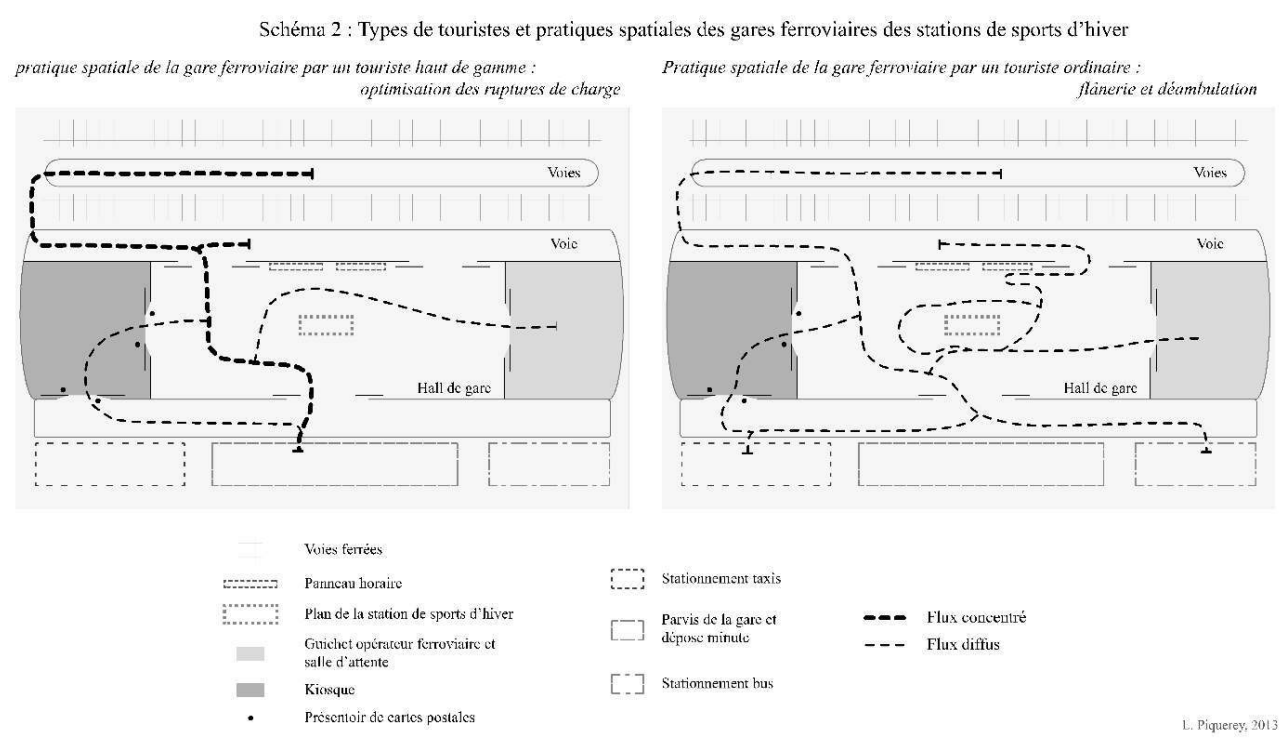

L. Piquerey, 2013.

13 Le « temps disponible », période entre la descente du train et la sortie de la gare, n'est pas un «temps mort » du déplacement, mais une phase de «déambulation ». Le hall de gare est aménagé de telle sorte que les individus puissent dans un premier temps «s'imprégner » de la destination touristique, avec l'utilisation de symboles touristiques du lieu. La gare de Zermatt par exemple, semi-enterrée, n'offre aucune vue sur la station pour les passagers qui arrivent à destination. Pour que les clients ne ressentent pas la distance entre le quai et la sortie de la gare comme un temps perdu ${ }^{1}$, mais bien comme les premiers moments du séjour, le mur principal de la gare est recouvert de traductions du mot «bienvenue» en plusieurs langues (ill. 1). Ces inscriptions permettent à la fois de mettre en avant le caractère international de la station et de toucher les clients en s'adressant à eux dès leur arrivée et dans leur langue maternelle. Après cette première phase de contact, suit une phase de découverte de la gare, nommée ici la « déambulation ». En effet, le touriste, n'ayant pas de repère présent dans l'espace, doit en identifier, notamment en utilisant ceux de son quotidien. Les guichets des opérateurs ferroviaires et les boutiques deviennent des repères qui répondront à ces attentes: s'informer sur la destination avec les plans présents dans le hall de gare, ou sur les trajets à venir au guichet de l'opérateur; se restaurer, acheter la presse ou simplement flâner dans les boutiques. Ce sont dans ces dernières que se trouvent les cartes postales, sur des 
présentoirs placés dans des lieux stratégiques: les portes d'entrées ou de sorties. Cette phase de déambulation permettra au touriste d'avoir au minimum dans son champ de vision les cartes postales, ce qui participera à la diffusion de l'image de la destination touristique, même s'il n'y a pas d'acte d'achat.

\section{1. Mur intérieur de la gare de Zermatt.}

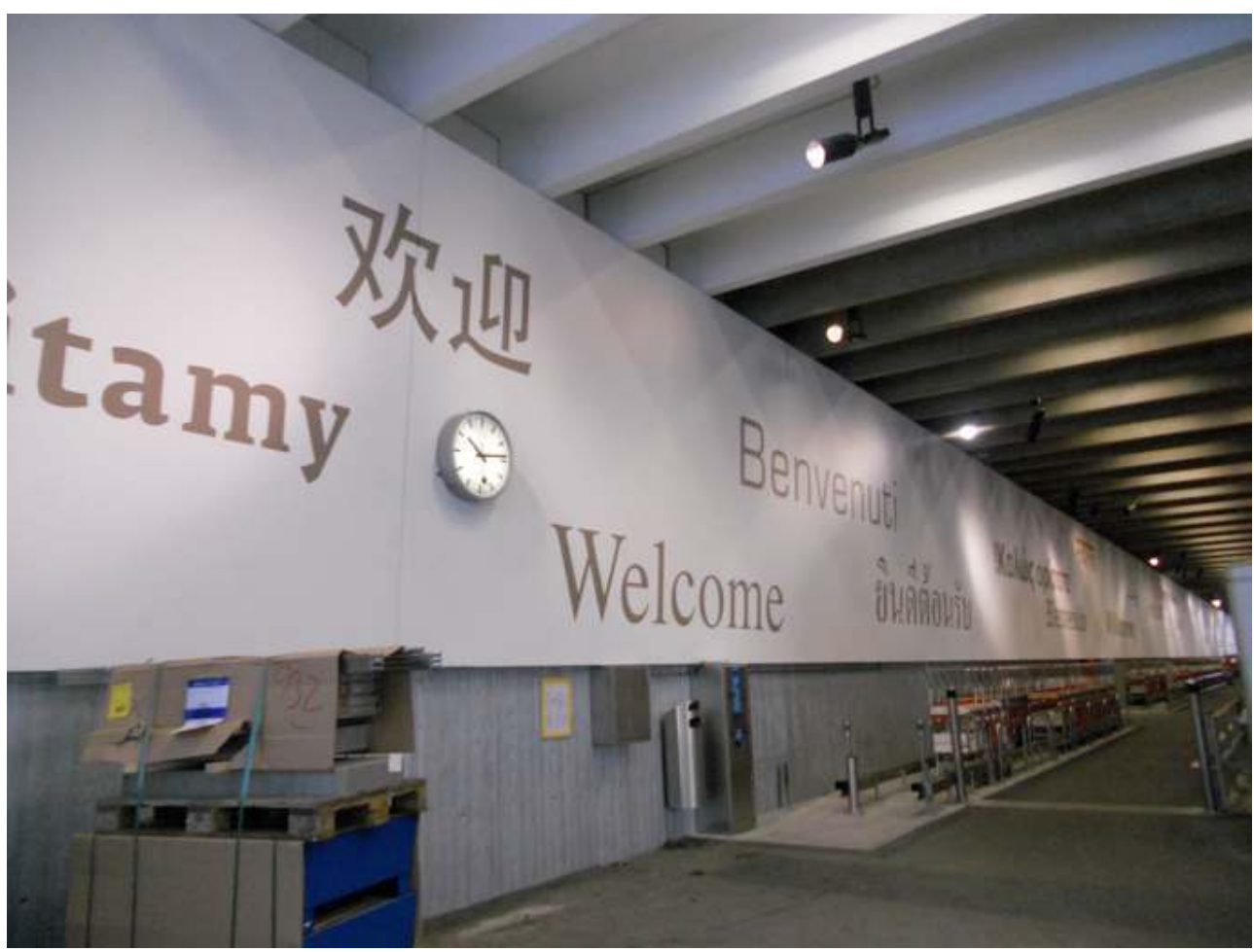

Une représentation du caractère international des clientèles de la destination touristique.

L. Piquerey, 2013.

Les lieux de vente des cartes postales au sein des stations de sports d'hiver haut de gamme, et notamment leur emplacement dans les gares ferroviaires, correspondent aux espaces que côtoient principalement les touristes des catégories socio-économiques moyennes, voire inférieures. Ces deux catégories seraient les clients ciblés par ce produit touristique. Cependant, nous pouvons noter que les cartes postales peuvent être dans certains cas exclusivement à destination des clientèles les plus aisées. Certains hôtels haut de gamme ou de luxe utilisent les cartes postales dans les brochures touristiques proposées à la clientèle; le recto est constitué d'une image à vue simple ou multiple de l'hôtel et le verso est laissé libre pour écrire le texte et l'adresse du destinataire. Nous ne savons pas si ces cartes postales seront envoyées par les clients, mais les images présentées mettent en avant les points forts des hôtels, participant ainsi à la diffusion de la montagnité à travers le filtre du luxe, avec la mise en scène par exemple de l'architecture, de la décoration des chambres et de l'environnement proche. Ces types de cartes postales sont dénuées de toutes traces humaines, pour permettre au client soit de se projeter dans l'hôtel, soit de garder une trace de son séjour. Cette seconde hypothèse est particulièrement mise en avant dans la brochure de l'hôtel The Omnia à Zermatt. Lorsque le client ouvre la brochure, des cartes postales de l'hôtel sont présentées dans un dépliant de couleur beige. Ce dernier s'apparente à une collection d'images où ce n'est pas la technique de la carte postale qui est mise en avant, mais plutôt l'idée d'une collection 
de souvenirs, d'un album d'images utilisant le format des cartes postales $(10,5 \times 14,8 \mathrm{~cm})$. À la différence des autres types de cartes, les cartes postales proposées dans les brochures des hôtels haut de gamme sont produites à des fins purement marketing, l'objectif étant de donner envie à la clientèle de venir ou de revenir séjourner dans l'hôtel.

D'une manière générale, les lieux de vente des cartes postales résultent d'une stratégie de maillage territorial des stations de sports d'hiver, établie dans un premier temps par les commerçants qui les vendent (choix du lieu d'implantation de la boutique) et par les éditeurs qui choisissent de proposer des cartes postales dans les lieux les plus visibles, créant ainsi une aire de vente de leurs produits notamment en se spécialisant dans certains types de cartes postales (par exemple, l'éditeur EDY en produit uniquement sur le thème de la montagne). Les offices de tourisme, créateurs de la communication touristique des destinations, tirent également profit des cartes postales, car elles sont des vecteurs de l'image des stations de sports d'hiver. Les liens entre les éditeurs et les décideurs de la communication touristique des stations de sports d'hiver dans la production d'images touristiques n'ont pu être établis ; cependant, en échangeant avec des propriétaires de points de vente de cartes postales, il semble que celles qui sont proposées résultent d'un consensus entre les meilleures ventes des éditeurs et les goûts personnels des gérants. Les cartes postales, à travers leurs «visées commerciales et promotionnelles » (Hossard, 2005, p. 12), participent donc, au même titre que les brochures touristiques officielles, à la construction de la renommée des stations de sports d'hiver haut de gamme.

Toutefois, la distribution des lieux de vente n'est-elle pas également représentative de la ségrégation socio-spatiale présente entre les différents types de clientèles au sein des stations de sports d'hiver de l'échantillon d'étude? Les points de vente des cartes postales recensés correspondent aux zones les plus touristiques, ayant la fréquentation la plus élevée. Les cartes postales, par leur faible coût et les images associées, apparaissent comme des produits touristiques de masse, à destination du plus grand nombre en raison de leur faible coût d'achat, et standardisés. Cette fréquentation se traduit par une image plutôt homogène des espaces touristiques, vectrice de la montagnité. Certaines cartes postales sont ainsi créées pour pouvoir être transposées dans plusieurs espaces touristiques, sans changer l'image mais simplement le (ou les) toponyme(s), ce qui réduit les coûts de production des éditeurs. La maison d'édition André, basée à Grenoble, a édité une série de cartes postales à l'imagerie " ancienne ", mais qui sont en réalité des copies de la symbologie utilisée dans deux affiches commandées à deux illustrateurs (R. Broders et $\mathrm{H}$. Reb) par la compagnie des chemins de fer de Paris à Lyon et à la Méditerranée (PLM) au début $\mathrm{du} \mathrm{xx}^{\mathrm{e}}$ siècle. Ces cartes postales permettent de représenter la tradition touristique du lieu et ainsi d'affirmer la capacité de la destination à rester un espace touristique malgré le (ou grâce au) temps passé (ill. 2 et 3). Si celle de Megève et du Vald'Arly rappelle le lancement du tourisme dans la vallée ${ }^{2}$, celle de Courchevel est clairement une "réécriture historique" ayant pour but de légitimer une identité ancienne et traditionnelle liée à la pratique des sports d'hiver et au tourisme au sein de la destination ${ }^{3}$. 
III. 2 et 3. L'utilisation de l'imagerie ancienne dans les cartes postales, symbole de la montagnité des lieux?

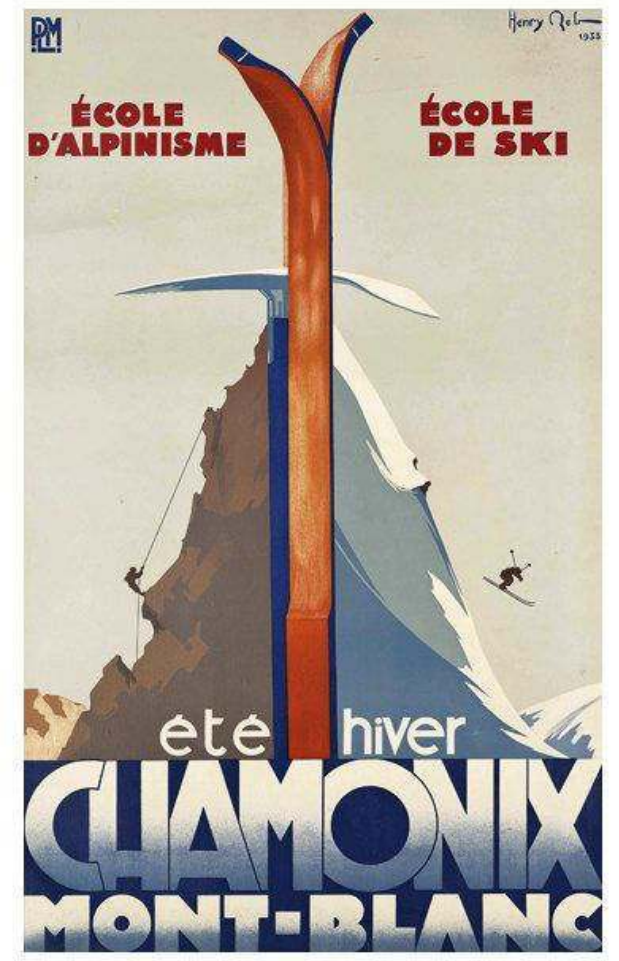

III. 2. Affiche réalisée par H. Broders et H. Reb, début du Xxe siècle.

Musée alpin Chamonix.
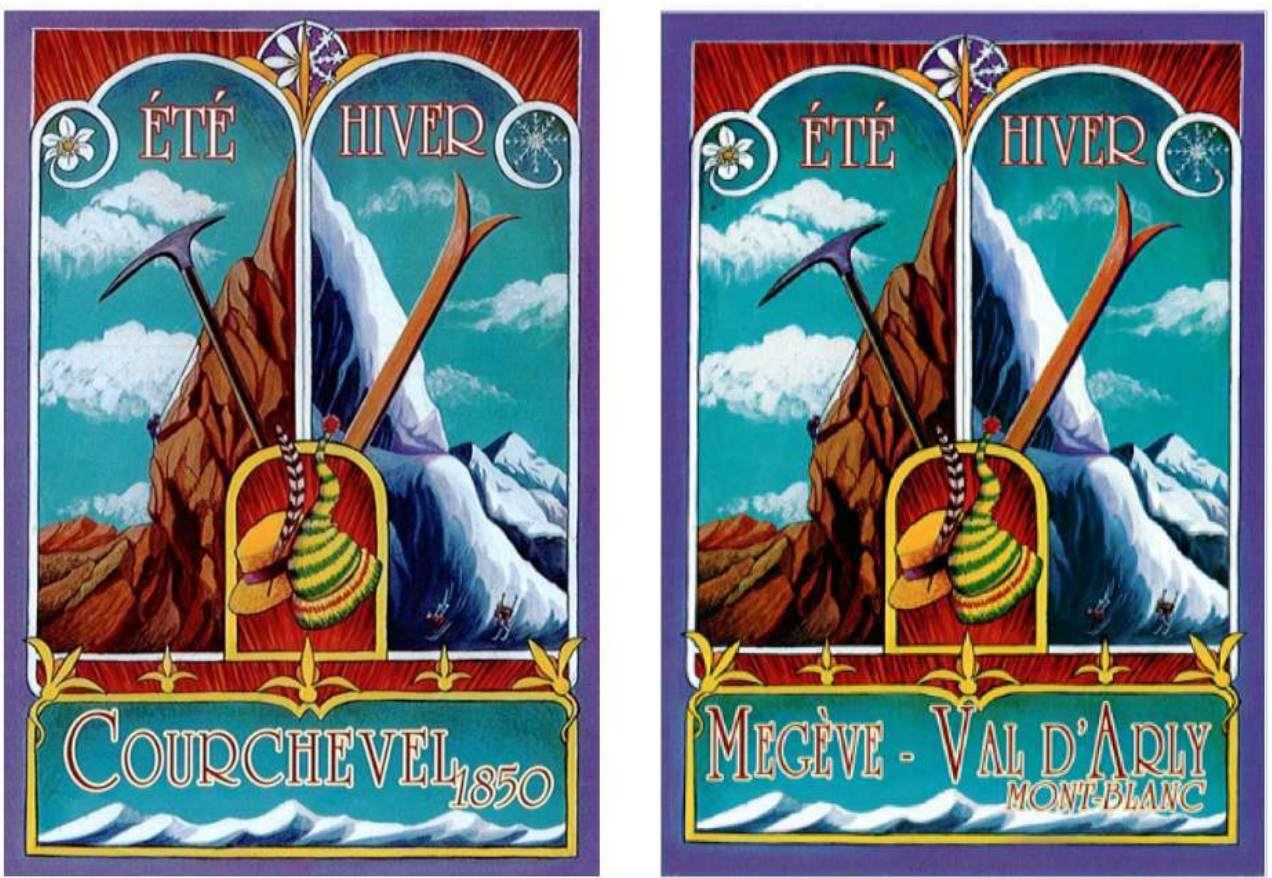

III. 3. Cartes postales éditées par la maison d'édition André, 2010.

17 Cependant, des rééditions d'affiches anciennes en format carte postale sont proposées par des éditeurs français ou suisses. Elles ont été produites entre 1880 et 1940 directement 
par les stations de villégiature prisées à l'époque, ou par des compagnies ferroviaires (PLM ; Gornergrat ; Compagnie des Rhätische Bahn pour la promotion du Glacier Express). Les destinations touristiques correspondent aux stations haut de gamme de l'échantillon d'étude présenté précédemment, au-dessus desquelles trône Chamonix. Les compagnies ferroviaires ont été des acteurs du développement touristique de ces stations puisque, pour commercialiser les billets entre les grandes villes et les stations de villégiature, elles choisissent de fonder leur communication sur l'image de la destination, la clientèle faisant ainsi abstraction du temps et du coût du déplacement en se projetant dans l'affiche (Sutton, 2008). Ces cartes postales vendues dans les kiosques des gares permettent à la destination d'intégrer son caractère mondain, élitiste, dans des points de vente standardisés. Une étude des affiches anciennes des stations suisses de l'échantillon, rééditées par l'éditeur Photoglob (Zurich) dans le cadre d'une exposition d'affiches anciennes de stations de villégiature suisses au Musée des arts et métiers de Zürich, permet de dater du début du $\mathrm{xx}^{\mathrm{e}}$ siècle la promotion du tourisme hivernal pour Davos et Saint-Moritz, au début des années 1930 pour Zermatt et à partir de 1940 pour CransMontana (Gstaad est absente de la collection). Les affiches mettent en scène aristocrates et bourgeois (reconnaissables par leur tenue vestimentaire) s'adonnant aux sports hivernaux de glisse: ski, patinage, luge. Les personnages se voulant représenter les «montagnards » sont représentés uniquement sur les affiches faisant la promotion de la saison estivale de Saint-Moritz, sous les traits de jeunes femmes souriantes, vêtues de tenues « traditionnelles ».

18 Les affiches estivales ont une construction identique, quelle que soit la station représentée : en bas de l'affiche, un encart sur fond blanc ou une couleur claire contient le nom de la station, le canton, et l'altitude. Cette dernière indication ne sert pas seulement à donner une information sur la localisation de la station, mais à justifier sa qualité de vie et notamment la qualité de l'air, à une époque où les stations mondaines de villégiature estivale sont aussi des stations climatiques ayant des vertus médicinales (présence de sanatoriums). Certaines affiches estivales des stations présentes dans cette collection illustrent le passage de la pratique curative et de l'héliothérapie au sein des stations à la pratique du « bon air » quotidien par les villégiateurs du début du $\mathrm{xx}^{\mathrm{e}}$ siècle (Lüthi, 2005). Les affiches se composent de trois parties : au premier plan se trouvent des individus qui pratiquent la promenade, la contemplation du paysage ou le golf (un quart de l'affiche), au second plan le lac (aménité très fréquemment présente dans les stations climatiques du XIX ${ }^{e}$ siècle) au bord duquel s'est développée la station, représentée par des bâtiments plus ou moins proches du premier plan (un autre quart de l'affiche). Enfin, en arrière-plan l'environnement proche de la station : la haute montagne, avec ses sommets coiffés par les «neiges éternelles» (la moitié restante de l'affiche) (ill. 4). Les rééditions d'affiches anciennes instaurent donc une standardisation de l'histoire de ces destinations haut de gamme avec des images reflétant les traditions de villégiature de la bourgeoisie et de l'aristocratie. En dehors de cette collection, d'autres éditeurs éditent des affiches anciennes, ou des images utilisant les codes de ce type de représentation. 
III. 4. Reproduction format carte postale d'une affiche faisant la promotion de Saint-Moritz (1930).

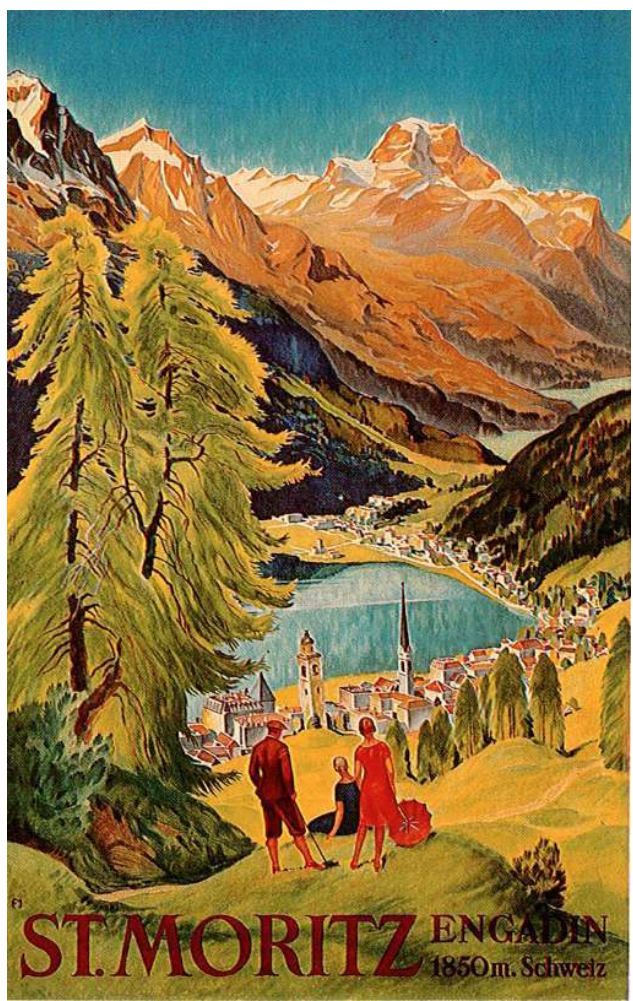

Édition Photoglob, collection du Musée des arts et métiers, Zurich, 2010.

Ainsi, il apparaît que l'imagerie diffusée par les cartes postales légitime le caractère haut de gamme des stations de l'échantillon, soit en rééditant des affiches anciennes, qui étaient déjà vectrices d'une image touristique élitiste dès leur création, soit en construisant "l'histoire du lieu», validant sa tradition touristique. La représentation d'une station touristique pourrait donc se définir comme l'agencement et la mise en scène des éléments socio-spatiaux présents dans une destination à des fins de diffusion touristique. Les cartes postales transforment ainsi le touriste acheteur en touriste acteur de communication, car en envoyant une carte postale il diffusera à son tour la montagnité du lieu. Les présentoirs des cartes postales, à travers leur localisation, servent à cette construction de l'identité d'une station par l'image, en occupant des lieux visibles par le plus grand nombre.

\section{Image et identité spatiale : le rôle des cartes postales dans la communication touristique de Megève}

Cette étude exploratoire sur le rôle des cartes postales dans la diffusion de l'image haut de gamme des stations de l'échantillon d'étude a pour objectif dans un premier temps de questionner la construction des symboles socio-culturels de la montagnité par l'analyse des types de cartes postales présentes au sein des stations de sports d'hiver, construisant, ou non, une image d'exclusivité du lieu. Pour répondre à cette problématique, une analyse des cartes postales vendues dans la station de Megève a été réalisée dans l'objectif de mettre en avant les images clés, vectrices d'une montagnité mégevanne. 
21 Dans un premier temps, trois points de vente ont été sélectionnés, représentant chacun un des types de commerces proposant des cartes postales : la boutique de souvenirs Aux Nevès située en face de l'office de tourisme, à l'extrémité sud-ouest de la principale rue touristique; une Maison de la presse située à l'extrémité nord-est de la principale rue touristique ; un Petit Casino, situé sur la route nationale à l'entrée de la commune. Dans chaque point de vente choisi, l'ensemble des présentoirs de cartes postales ont été photographiés et un court entretien a été réalisé avec le gérant du commerce pour connaître les critères de sélection des cartes postales.

Une grille d'analyse, basée sur trois critères, a été appliquée au matériel collecté :

- Contexte général de la carte postale : lieu de vente ; époque de publication de la carte postale.

- Organisation de la carte postale : vue simple ou vue multiple ; saison; période de la journée ; éléments au premier plan ; éléments en arrière-plan ; part de ces éléments dans l'occupation de la surface de la carte postale.

- Éléments mis en scène dans la carte postale : référence au " patrimoine territorial » (éléments naturels ou anthropiques institutionnalisés comme des emblèmes spatiaux); personnage(s) (homme ou femme, époque, action dans la carte postale) ; activités sportives (ski, patinage, bobsleigh, polo sur neige, randonnée, promenade, golf, alpinisme, polo) ; représentation de la station (chalets dispersés, village groupé, infrastructure de loisirs).

Au total, 478 cartes postales, vendues à Megève, ont été analysées. Dans un premier temps, nous pouvons mettre en avant l'omniprésence de deux toponymes au sein des cartes postales : Megève (36 \%) et mont Blanc (7 \%). Les cartes à vues multiples de Megève et les cartes à vue simple utilisent principalement des images «clés » pour présenter la station : place de l'Église, rue Monseigneur-Conseil (ruelle du cœur historique de Megève avec un pont en pierre) (ill. 5). Les vues d'ensemble de Megève présentent le mont Blanc en arrière-plan dans la majorité des cas ; a contrario, le mont Joly et le mont d'Arbois, emblématiques du développement des sports d'hiver à Megève avec le téléphérique de Rochebrune mis en service en 1933, et l'hôtel du Mont d'Arbois créé par la famille Rothschild, apparaissent seulement sur une carte postale. Les attributs de la station s'éclipsent donc derrière des attributs de plus grande notoriété, et plus génériques. 


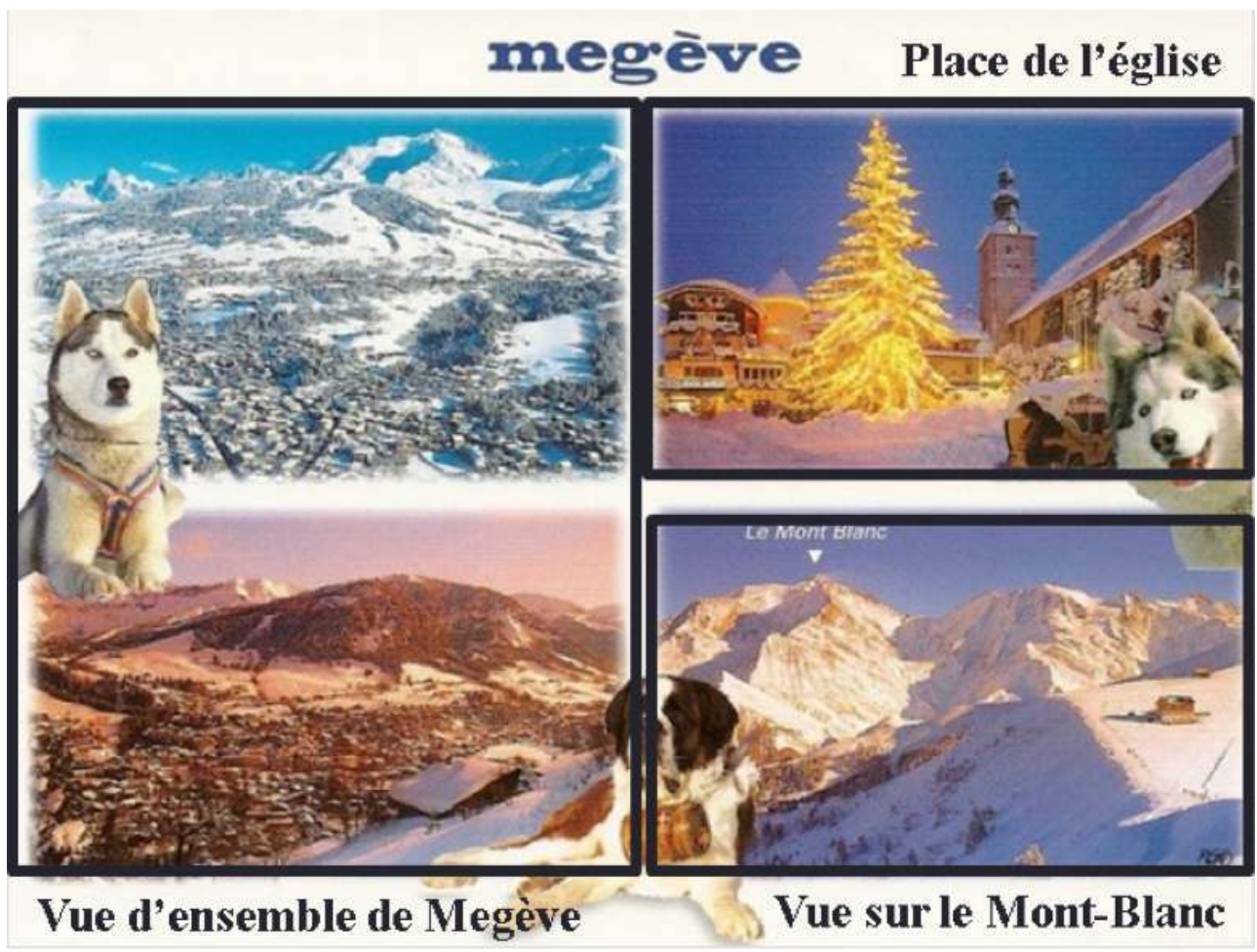

Carte postale à vues multiples, maison d'édition André, 2011.

Les cartes à vues multiples présentent aussi le mont Blanc et le village de Megève, associant ainsi les deux entités par l'image et parfois également en juxtaposant les toponymes. Ce type de construction n'est pas récent, puisque dès les années 1940 des cartes présentant « Megève et le mont Blanc » sont éditées par la maison d'édition Cellard (Lyon). Le mont Blanc est représenté sur environ $20 \%$ des cartes postales à vue simple et vues multiples de l'échantillon, certaines cartes postales étant construites par exemple avec un coucher de soleil sur les cimes du mont Blanc, sous lequel est inscrit Megève. Le val d'Arly, vallée dans lequel est inscrite la commune de Megève, n'est pas représenté dans l'imagerie des cartes postales de l'échantillon, et le toponyme " val d'Arly » apparaît seulement dans cinq cartes postales.

Les sports d'hiver, et notamment le ski, sont présents sur les cartes postales avec la pratique du ski. Les remontées mécaniques sont visibles sur $1 \%$ des cartes postales : une carte laisse apparaître des pylônes et des câbles, et six cartes montrent une image avec un téléski (non identifié sur Megève), toujours identique, avec une localisation similaire sur toutes les cartes postales. Or, Megève est une station qui a fondé son modèle de développement sur le ski, dès 1919. Les tracés et les infrastructures des remontées mécaniques sont absents des cartes postales à vue large de la station, elles ont donc soit été évitées lors de la prise de vue, soit gommées par des logiciels au moment de la mise en forme, ce qui peut paraitre curieux pour une carte postale d'une station de sports d'hiver.

Sur les cartes de type « ancien » ou utilisant les codes des anciennes cartes postales grâce au sépia ou au noir et blanc, les pratiquants de ski sont représentés en tenue mondaine, il en est de même pour les affiches « anciennes ». Seules deux cartes « anciennes » ont pour 
sujet des scènes de la vie paysanne. La difficulté à dater ces deux cartes ne nous permet pas d'affirmer leur authenticité, mais quoiqu'il en soit elles semblent assez caricaturales.

Près de $16 \%$ de l'échantillon est constitué de cartes humoristiques, érotiques ou d'animaux; avec les cartes postales de paysages de montagne sans localisation précise, elles forment la majeure partie des cartes transposables dans d'autres destinations touristiques de montagne. Les cartes « anciennes », représentent 11,5\% de l'échantillon, et $50 \%$ d'entre elles représentent des pratiquants de sports d'hiver, sans indication ou détail permettant d'identifier le lieu ou l'époque de la prise de vue, et sont donc également facilement transposables. Ces cartes transposables ne représentent pas la montagnité de Megève, mais une montagnité standardisée, construite par l'utilisation de symboles se voulant représentatifs de l'espace montagnard dans l'imaginaire populaire (carte postale humoristique des marmottes ; image des chalets et des sapins enneigés) ou de façon plus localisée (carte des aiguilles du massif du Mont-Blanc, carte postale gastronomique utilisant la recette de la " croziflette $4^{4}$ ). La neige et les jeux de lumière, artificielle ou naturelle, sont les deux principaux outils des cartes postales qui permettent de mettre en forme l'aspect "cocon », en dehors du temps, que l'office de tourisme de Megève développe dans sa stratégie de communication.

À travers l'étude de cet échantillon de cartes postales, nous pouvons définir une carte postale type de la représentation d'une montagnité mégevanne :

- La zone inférieure de la carte postale serait laissée libre sur environ un centimètre. Il y aurait inscrit « Megève - mont Blanc », sur un fond blanc.

- Dans la zone supérieure serait positionnée l'image, avec au premier plan une bande de neige fraîche sur laquelle serait placé un traîneau tiré par deux chevaux. Ensuite au second plan la place de l'Église de Megève entourée de sapins et en arrière-plan le mont Blanc.

Ainsi, les cartes postales vendues à Megève sont représentatives du lieu avec l'utilisation du mont Blanc comme emblème territorial, bien que la face sud-ouest soit moins utilisée que la face nord dans l'imagerie populaire, et avec l'image du village (place de l'église) qui est omniprésente. Le caractère haut de gamme, tant mis en avant dans les brochures touristiques de station, n'apparaît qu'à travers des ambiances ou des détails (pratique des sports d'hiver par les catégories socio-économiques supérieures dans les cartes «anciennes»). D'autres éléments, représentatifs du caractère haut de gamme de la destination dans les brochures, comme la gastronomie et les hôtels cinq étoiles de la communication " officielle », ne sont pas représentés, ils semblent même s'opposer aux produits gastronomiques régionaux et aux chalets en bois des alpages des cartes postales.

\section{Conclusion : la carte postale, une montagnité standardisée}

L'étude des cartes postales de Megève et de celles collectées lors des missions de terrain dans les autres stations de l'échantillon permet d'affirmer deux caractéristiques du rôle des cartes postales dans les stratégies de communication des stations haut de gamme de l'arc alpin. Tout d'abord, les éléments créant le caractère montagnard des espaces, la montagnité, sont utilisés par l'ensemble des éditeurs de cartes postales, qu'ils soient suisses, français ou autrichiens. Les sapins, la neige "fraîche », les "villages » cœurs des stations et les chalets sont autant d'éléments qui permettent à la destination touristique d'amorcer et de prouver sa montagnité, à un public qui lui aussi reçoit de la part d'autres 
acteurs touristiques des images standardisées des stations de sports d'hiver, via par exemple les brochures touristiques des tour-opérateurs ou promoteurs immobiliers. Ensuite, l'ensemble des cartes postales collectées émettent l'idée d'une contradiction entre les stratégies touristiques "officielles" des stations de sports d'hiver haut de gamme et l'image véhiculée par le biais des cartes postales. Si les cartes postales anciennes, issues de rééditions d'affiches ou non, sont des vecteurs du caractère élitiste des stations de sports d'hiver à travers les types d'activités choisis et la mise en scène de leurs pratiquants, les cartes récentes sont standardisées, avec l'utilisation d'images identiques pour plusieurs cartes postales différentes (principalement des cartes postales à vues multiples), représentant d'abord le cadre naturel et le cadre anthropisé «traditionnel» de la station de sports d'hiver haut de gamme. Dans les cartes postales modernes, le caractère haut de gamme des stations de sports d'hiver de l'échantillon apparaît seulement à travers des éléments ponctuels, qui prendront sens uniquement pour les catégories socio-économiques supérieures, car ils font écho à leur espace vécu (prise de vue des hôtels haut de gamme et des palaces) ou à leurs pratiques (ill.6), considérées comme étant représentatives de pratiques de classe (Saint Martin, 1989) (carte postale des tournois de polo sur neige).

\section{6. Carte postale de Megève}

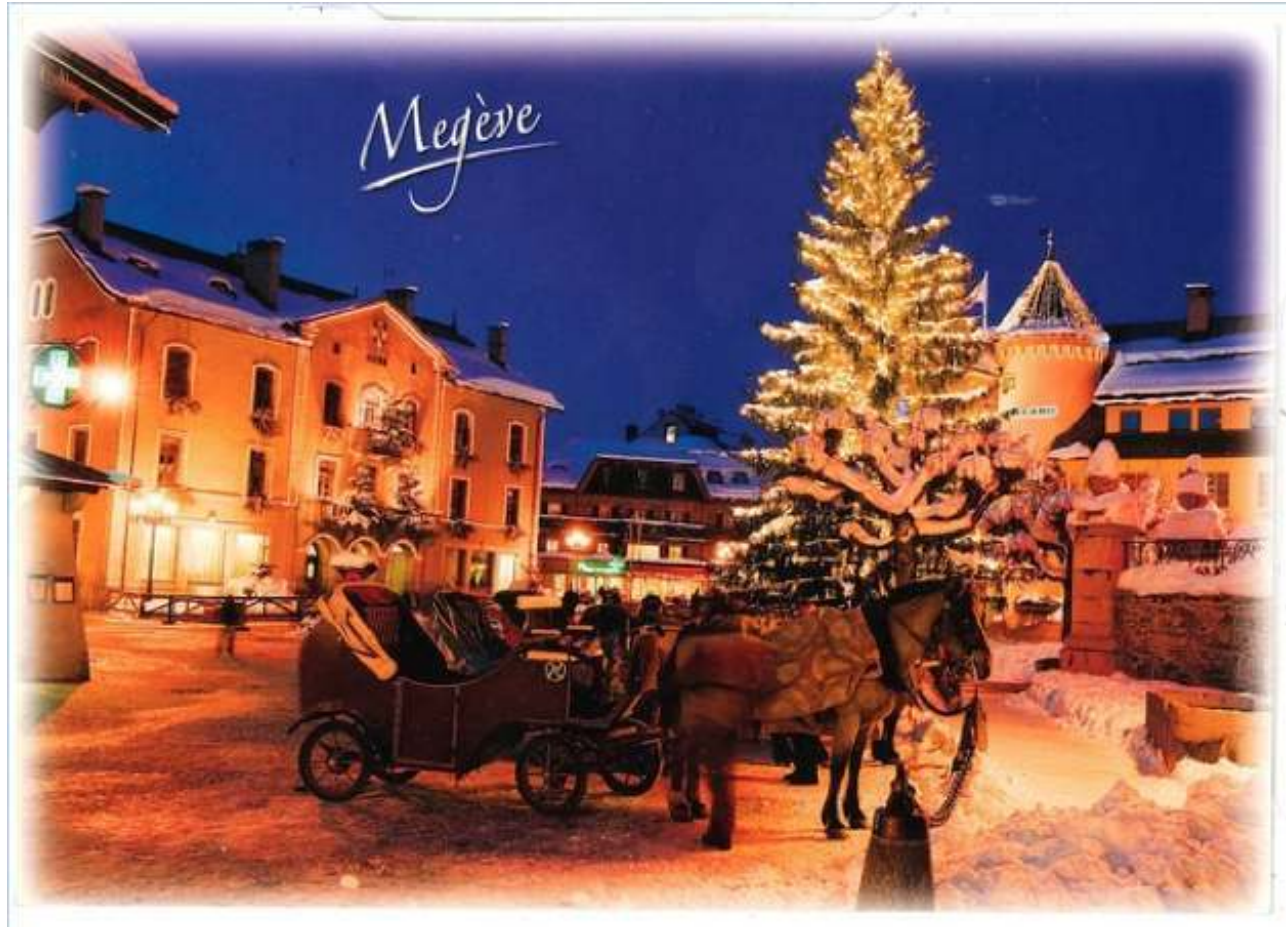

Photographie prise depuis la place de l'Église. Si cette carte postale souhaite diffuser l'image d'un village de montagne sous la neige, les détails de la prise de vue retranscrivent le caractère haut de gamme de la station : le sapin est serti de cristaux Swarowski et la tour en arrière-plan porte le patronyme Allard, maison de couture mégevanne, créatrice notamment du fuseau de ski en 1930. Maison d'édition EDY, 2010.

31 À travers les arguments avancés dans cet article, une question émerge : les cycles de vie des cartes postales ne seraient-ils pas standardisés, avec une imagerie de la montagnité globale, des lieux de ventes visibles et fréquentés par le plus grand nombre, et à destination d'un public ayant une image de la montagne uniformisée ? L'étude des cartes 
postales montre que la montagnité est une notion dont les fondements sont constitués à partir des images et des codes incorporés dans l'imaginaire socio-culturel de la montagne par l'ensemble des individus, mais dans laquelle des variations sont perceptibles notamment en fonction des caractéristiques socio-spatiales de chacun.

\section{BIBLIOGRAPHIE}

Antoine S. Bailly, Robert Ferras et Denise Pumain (dir.), Encyclopédie de géographie, Economica, 1999.

Roland Barthes, « Rhétorique de l'image », Communications, vol. 4, n 1, 1964, p. 40-51.

Jean-Paul Bozonnet, Des monts et des mythes. L'imaginaire social de la montagne, « Montagnes ", Presses universitaires de Grenoble, 1992.

Bernard Debarbieux, « Construits identitaires et imaginaires de la territorialité : variation autour de la figure du "montagnard" ", Annales de géographie, nº 660-661, 2008, p. 90-115.

Isabelle Frochot et Patrick Legohérel, Le Marketing du tourisme, Dunod, 2007.

Anne Gilbert, « L'idéologie spatiale : conceptualisation, mise en forme et portée pour la géographie?", Espace géographique, vol. 15, nº 1, 1986, p. 57-66.

Nicolas Hossard, Recto-verso, les faces cachées de la carte postale, Arcadia, 2005.

Yvan Hostettler, Cervin, montagne de pub, Olizane, 1990.

Yvan Hostettler, Cervin, top model des Alpes, Olizane, 2006.

Jacques Lévy et Michel Lussault (dir.), Dictionnaire de la géographie et de l'espace des sociétés, Belin, 2003.

Jean-Baptiste Litot, Paysage et médiatisation dans les Alpes françaises. Approche géographique de la diffusion des cartes postales paysagères, thèse de doctorat en géographie, université de FrancheComté, 2010.

Dave Lüthi, «L'influence du bon air sur l'architecture. Une "guérison formelle" ? Apparition du sanatorium alpin en Suisse, 1880-1914», Revue de géographie alpine, $\mathrm{n}^{\circ}$ 1, 2005, p. 43-52.

Serge Paugam (dir.), Les 100 mots de la sociologie, « Que sais-je ? », Presses universitaires de France, 2010.

Le Petit Robert de la langue française, Le Robert, 2011.

Michel Pinçon et Monique Pinçon-Charlot, « L'aristocratie et la bourgeoisie au bord de la mer. La dynamique urbaine de Deauville », Genèses, nº 16, 1994, p. 69-93.

Michel Pinçon et Monique Pinçon-Charlot, Les Ghettos du Gotha : comment la bourgeoisie défend ses espaces, « Points », Le Seuil, 2007.

Lise Piquerey, Entre fermeture et visibilité. La ségrégation et l'entre-soi dans les stations de sports d'hiver en Savoie et en Haute-Savoie, mémoire de master 2 recherche en géographie, université de Savoie, 2012. 
Monique de Saint Martin, «La noblesse et les "sports" nobles ", Actes de la recherche en sciences sociales, vol. 80, 1989, p. 22-32.

Kevin Sutton, «Traverser les Alpes par les affiches », dans Gilles Fumey, Jean Varlet et Pierre Zembri (dir.), Mobilités contemporaines. Approches géoculturelles des transports, Ellipses, 2008,

p. 191-203.

\section{NOTES}

1. Zermatt est une station piétonne, où la quasi-totalité des départs et des arrivées des clientèles se font en train depuis le Matterhorn Terminal de Täsch.

2. Quand bien même Megève n'a jamais été un centre d'alpinisme, contrairement à ce que laisse suggérer le piolet.

3. Megève connaît sa première saison hivernale pendant l'hiver 1913-1914 sous l'impulsion de la baronne Noémie de Rothschild, qui selon la légende ne souhaitait plus côtoyer des individus germanophones pendant ses séjours de villégiature, notamment à Saint-Moritz, en raison du climat géopolitique de l'époque en Europe. Courchevel 1850 est une station de seconde génération, construite à partir de 1946, sous l'impulsion du ministère de la Reconstruction et du conseil général de la Savoie.

4. La croziflette est un plat récemment élaboré en Savoie. La recette suit celle de la tartiflette en remplaçant les pommes de terre par des crozets, qui sont des pâtes au sarrasin produites localement.

\section{RÉSUMÉS}

Cet article vise à questionner le lien entre les cartes postales et les stations de sports d'hiver haut de gamme de l'arc alpin. En effet, l'exclusivité de ces lieux semblent de premier abord s'opposer à la capacité de diffusion aussi bien spatiale que sociale des cartes postales. Il apparaît toutefois que les cartes postales participent au caractère élitiste de ces stations touristiques, en présentant notamment des scènes de vie mondaine, héritage de la villégiature du xix ${ }^{e}$ siècle. Le rôle des cartes postales au sein de la communication touristique, peu étudié au sein du marketing, semble ainsi être un vecteur de l'image des stations de sports d'hiver haut de gamme. De plus, elles participent à la création de la "montagnité ", imaginaire se voulant être le plus représentatif possible des espaces montagnards en faisant appel à des codes sociaux inscrits dans la pensée collective.

This article aims to question the link between postcards and alpine winter sports resorts. Indeed, the exclusivity of these places seems at first sight to oppose the spatial as well as social diffusion capacity postcards. It appears however that the postcards to the elitist part of these resorts, including presentation of scenes of fashionable life, legacy of the nineteenth century resort. The role of postcards in the tourist communication, little studied in the marketing seems to be a vector image of the winter sports resorts. In addition, they participate to create the "montagnité" imaginary wanting to be as representative as possible of mountain areas by using social codes register in the collective thought. 
INDEX

Mots-clés : carte postale, station de sports d'hiver haut de gamme, arc alpin, imaginaire montagnard, idéologie spatiale, identité sociale

Keywords : postcard, winter sports resorts top of the range, Alps, mountain imaginary, space ideology, identity

\section{AUTEUR}

\section{PIQUEREY LISE}

Docteure en géographie, membre associée au laboratoire EDYTEM UMR 5204 - Université Savoie Mont Blanc. Elle a soutenu, en 2016 une thèse intitulée Goldens Snow. Ségrégation et entre-soi dans les stations de sports d'hiver haute de gamme en Autriche, en France et en Suisse. 